\title{
VIOLENCIA SEXUAL: CULPABILIDAD DE LOS VARONES Y MASCULINIDAD*
}

\author{
Sexual violence: \\ Guilty of males and masculinity
}

Ricardo RODRÍGUEZ LUNA

\begin{abstract}
Sumario:
Introducción I. Culpabilidad y violencias sexuales: cosa de hombres 1.1 Hallazgos estadísticos II. El Género masculino y la tradición criminológica 2.1. El positivismo: la esencialización de la sexualidad 2.2. La óptica sociológica: el afianzamiento de los roles socio-sexuales 2.3. El enfoque de género: hacia una perspectiva de lo masculino III. Comentario final Bibliografía.
\end{abstract}

Resumen. En este artículo se indaga en torno a los vínculos entre violencia sexual y género masculino. En primer lugar, se ilustra la dimensión de la culpabilidad de los varones de las violencias sexuales, lo anterior con base en algunos datos estadísticos relativos a procesados y sentenciados. A partir de ello, en segundo término, se analiza la forma en que la criminología ha tenido en cuenta el género masculino al explicar dichas violencias y en qué medida la ha vinculado a la sexualidad de los varones.

Palabras clave: violencias sexuales, varones, criminología, estadística criminal, masculinidad.

Abstract. This paper analyzes the possible links between sexual violence and male gender. First, based on statistical records, it shows the degree of male culpability in this kind of violence. From this, secondly, it is analyzed the way in which have taken into account the male gender, specifically, male sexuality, in criminological explanations about sexual violence.

Keywords: sexual violence, male, gender, criminology, masculinity, criminal statistics.

\section{Introducción}

El delito y la violencia son problemáticas de amplio alcance que lesionan la convivencia social en diversos sentidos, afectan a la población en general e inciden en el desarrollo económico y

\footnotetext{
* Este trabajo se ha desarrollado en el marco de la investigación intitulada "Adolescencia y violencia sexual en Guanajuato: retos y oportunidades en materia de seguridad y prevención delictiva"; aprobada en la Convocatoria Institucional de Investigación Científica (CIIC, 2019; convenio no. 086/2019) de la Dirección de Apoyo a la Investigación y al Posgrado. El autor agradece a la Universidad de Guanajuato el apoyo para la realización de este proyecto.

** Profesor-investigador. Departamento de Derecho de la División de Derecho, Política y Gobierno. Campus Guanajuato, Universidad de Guanajuato., e. mail: ricardo.rodriguez@ugto.mx
} 
político del país; y aunque hombres y mujeres somos víctimas de diversos tipos de delitos, algunos de estos afectan principalmente a uno u otro sexo.

Es el caso de las violencias sexuales, mismas que tienen especial impacto en la seguridad de las mujeres, pero, a su vez, todo parece indicar que casi de forma exclusiva los varones ejercen estas formas de violencias. ¿Por qué esto es así? ¿Por qué los varones ejercen violencias en altas proporciones?, son cuestionamientos que han preocupado a las ciencias jurídicas en general, en particular al derecho penal y la criminología, aunque también a disciplinas como la sociología, la antropología o la psicología.

En la presente investigación, desde una perspectiva socio-jurídica, se propone un enfoque que centra su atención en el género masculino y en la responsabilidad penal de las violencias sexuales. De manera particular se indaga la dimensión de la culpabilidad de los varones de las violencias sexuales, situación que se ejemplifica con algunos datos del sistema de justicia penal del estado de Guanajuato, México. A partir de ello se estudia la forma en que han abordado dicha problemática diversas perspectivas criminológicas, particularmente se analizan las relaciones entre delitos sexuales, género masculino y sexualidad de los varones. Se pretende así, en el marco de la teoría criminológica y de género, indagar en torno a los vínculos entre la responsabilidad penal de las violencias sexuales y la sexualidad masculina.

Para alcanzar dicho objetivo el presente trabajo está estructurado en dos apartados. En el primero, aunque de forma somera, se ilustra la culpabilidad de los varones de las violencias sexuales, para esto se emplean algunos datos estadísticos del sistema de justicia penal guanajuatense, en particular de hombres procesados y sentenciados. A partir de ello, en segundo lugar, se plantea la forma en que han explicado dicha violencia las vertientes criminológicas positivista, las teorías sociológicas y de la diferencia sexual. En cada una de estas, como antes se señaló, se analizan las relaciones entre delitos sexuales, género masculino y sexualidad de los varones.

Cabe tener en cuenta, a manera de precisión metodológica, que en este estudio la noción de violencia(s) sexual(es) se asume en sentido amplio y se emplea para hacer referencia al conjunto de conductas tipificadas que protegen la libertad sexual, pero también, a las diversas formas de violencia implícitas en tales ilícitos. Los tipos penales referidos se concretan en diversas conductas, en el caso del estado de Guanajuato, son la violación (bajo diversos supuestos), el abuso sexual, el acoso sexual, el estupro o el hostigamiento sexual ${ }^{1}$.

La información estadística que se presenta se obtuvo de los registros del Instituto Nacional de Estadística y Geografía (INEGI), esencialmente de las bases de datos del Censo Nacional de Impartición de Justicia Estatal y los Registros Administrativos en el Ámbito de Impartición de Justicia en Materia Penal ${ }^{2}$. El análisis que se propone aborda la culpabilidad de las violencias sexuales de los varones, aunque se realizan observaciones particulares relativas a la violación y el abuso sexual.

La información se centró en personas procesadas y sentenciadas, pero bajo diversos criterios de búsqueda, entre los que destacan: tipo de delito, fuero común, año y sexo. En relación con estas variables, es importante señalar que a lo largo de este artículo se hace referencia a diversos valores porcentuales, a fin de evitar reiteraciones, cabe decir que todos estos cálculos son propios, en caso de no ser así, se hará la anotación respectiva. El período que se analiza corresponde

\footnotetext{
1 Código Penal del Estado de Guanajuato (2019), Título Tercero, de los delitos contra la libertad sexual, [en línea], disponible en: https://portal.pgjguanajuato.gob.mx/PortalWebEstatal/Archivo/normateca/2.pdf, arts., 180-187d (consultado el 24 de agosto de 2019).

2 Instituto Nacional de Estadística y Geografía (INEGi), [en línea], disponible en: www.inegi.com.mx (consultado el 28 de agosto de 2019).
} 
a los años 2010 a 2017; este constituye el último año de información disponible conforme al sitio web del INEGI, al menos en lo que respecta al Censo Nacional de Impartición de Justicia Estatal y los Registros Administrativos en el Ámbito de Impartición de Justicia en Materia Penal ${ }^{3}$.

En esta investigación, como se ha indicado, se emplean diversos datos del estado de Guanajuato, sin embargo, la finalidad sólo es ejemplificar una problemática de amplio alcance que definitivamente requiere investigarse con mayor amplitud a nivel municipal, estatal, regional y nacional. Así mismo, desde el punto de vista que aquí se sostiene, es necesaria una perspectiva que indague en torno a lo masculino y que favorezca el desarrollo de estrategias que permitan la implicación de los varones en la lucha contra la violencia.

\section{Culpabilidad y violencias sexuales: cosa de hombres}

La culpabilidad de los varones de las violencias sexuales se ilustra en este apartado, para ello, en primer lugar, se hará referencia a los hombres considerados probables responsables y/o procesados, y sentenciados por ilícitos de carácter sexual, aunque de manera particular, se realizarán algunos señalamientos en torno a los delitos de abuso sexual y violación; lo anterior, con base en algunos datos estadísticos del sistema de justicia penal del estado de Guanajuato. Mostrados estos datos, en segundo lugar, se realizarán algunas observaciones relativas a la información estadística reportada por las autoridades e instituciones respectivas.

En primer término, a manera de contexto, cabe hacer una breve referencia a las violencias sexuales en la República Mexicana; el contexto es tajante: los varones fueron sentenciados en el período 2010-2017, en promedio, en poco más del $97 \%{ }^{4}$ de los casos de delitos sexuales 5 . Esta

3 En la página web del INEGI fue posible leer, hasta el mes de julio de 2019, que la última actualización de la información se llevó a cabo el "26 de octubre de 2018 " y que la próxima se realizaría el " 25 de octubre de 2019 ". No obstante, a lo largo de esta investigación, se consultó información durante los meses de abril, mayo y junio; pero, el mes de julio dicha información cambió sin los avisos respectivos. Fue en el mes de agosto que se realizaron diversas aclaraciones sobre algunas actualizaciones, así mismo, el día 14 de agosto del 2019, el INEGI dio a conocer resultados preliminares del décimo Censo Nacional de Gobierno, Seguridad Pública y Sistemas Penitenciarios Estatales (CNGSPSPE), así como de la novena edición del Censo Nacional de Impartición de Justicia Estatal (CNIJE). Estos datos no son tenidos en cuenta en esta investigación por dos motivos principales, por un lado, porque constituyen información "preliminar" y esta fue publicada cuando ya había presentado una primera versión de este trabajo para su respectivo dictamen. Por otro lado, porque a lo largo de esta investigación, a pesar de las advertencias sobre las fechas previstas en la página electrónica del INEGI, se observaron algunas actualizaciones realizadas sin los avisos oportunos, por este motivo, se considera que dicha información "preliminar" es aún susceptible de nuevas actualizaciones. Instituto Nacional de Estadística y Geografía (INEGI), Censo Nacional de Impartición de Justicia Estatal 2018, [en línea], disponible en: https://www.inegi.org.mx/programas/ cnije/2018/default.html\#Documentacion (consultado el 28 de agosto de 2019).

${ }^{4}$ Este valor se obtuvo con base en aquello casos en los que sí fue identificado el sexo de las personas, ya que en algunos años se reportaron diversos porcentajes de personas cuyo sexo no fue identificado. Este valor alcanzó el 2012 un valor de $18.65 \%$, el 2014 fue de $10.96 \%$ y el 2017 de 17.02\%. Para cada uno de estos años ver la edición del Censo respectiva. Instituto Nacional de Estadística y Geografía (INEGI), Censo Nacional de Impartición de Justicia Estatal 2013, [en línea], disponible en: https://www.inegi.org.mx/programas/cnije/2013/ (consultado el 27 de agosto de 2019).

5 El INEGI reportó los siguientes delitos sexuales para los años 2010-2012: abuso sexual, violación simple e incesto; para el 2013, además de los anteriores: acoso sexual, hostigamiento sexual, violación equiparada y otros delitos sexuales; para el 2014, además de los anteriores: estupro, "otros delitos sexuales sin realización de cópula" y "otros delitos sexuales con realización de cópula"; estos dos últimos delitos no se reportan el 2015 ni el 2016. Sin embargo, el 2017, además de los anteriores, se reportan los delitos de "violación equiparada a menores", "otro tipo de violación" y "violación no especificado". La información de 2010-2012 proviene de: InstiTuTO NACIONAL DE Estadística y Geografía (INEGI), [en línea], disponible en: https:/www.inegi.org.mx/programas/judicialespenal/default.html\#Tabulados (consultado el 23 de julio de 2019). La información de 2013 a 2017 proviene, res- 
tendencia se confirma si se centra la atención en algunos ilícitos el abuso sexual y la violación (simple), e incluso se ve superada, ya que, en ambos, en un período de tiempo mayor, del 2000 al 2017, en promedio, en poco más del $98 \%{ }^{6}$ de los casos los varones fueron sentenciados.

En el estado de Guanajuato la información en torno a los delitos sexuales ${ }^{7}$ muestra una situación similar. En el período 2010 a 2017, en promedio, los hombres fueron procesados en un $87.68 \%{ }^{8}$. Los datos son elocuentes si la atención se centra en algunos delitos en particular: en el delito de abuso sexual, los hombres resultaron presuntos y/o procesados, en promedio, en un 98.78\%; un $96.84 \%$ en lo relativo a la violación. Es conveniente señalar que en ambos ilícitos únicamente se han tenido en cuenta aquellos casos en los que fue identificado el sexo de la persona, puntualización que resulta necesaria, ya que, el 2013, en lo relativo al abuso sexual se reportó en un $87.70 \%$ el sexo de las personas como no identificado; en el delito de violación esta proporción ascendió al $75.53 \%$.

En el ámbito judicial, en el período que se comenta ${ }^{10}$ y en cuanto al conjunto de delitos sexuales, los varones fueron sentenciados en un $\mathbf{9 9 . 1 6 \%}$ de los casos; estas alcanzaron un valor de $98.90 \%$ en cuanto al abuso sexual y $99.37 \%{ }^{11}$ en el caso de las sentencias por delito de violación. En lo que respecta a este ilícito es importante tener en cuenta que el 2017 no se reportó ninguna sentencia de violación simple, aunque se reportaron 124 personas procesadas, de las cuales 121 fueron varones. No obstante, a su vez, se informó de 9 sentencias por otro tipo de violación, sobre un total de 25 personas procesadas, de las cuales 24 eran hombres; así como también 143 sentencias de violación no especificado, 142 de ellas dictadas contra varones, pero, en este caso, sólo se reportaron 2 personas procesadas por este delito ${ }^{12}$.

En cuanto a las mujeres procesadas por delitos sexuales, en el período 2010-2017, el número de ellas se incrementó de forma drástica con posterioridad al 2013, de hecho, poco más del $89 \%$ del total fueron procesadas en los años 2014-2017; por mucho, 2015 fue el más numeroso de la década con veintidós mujeres procesadas por delitos sexuales. No obstante, el promedio de sentenciadas por tales delitos a lo largo de esta década se ha mantenido por debajo del $1 \%$.

Estos datos ilustran, entre otras cosas, el grado de culpabilidad de los varones en el ámbito de las violencias sexuales. La (des)proporción de esta culpabilidad, así como de (ir)responsabilidad social es contundente: de cada 10 delitos que atentan contra libertad sexual, (poco más de) 9

pectivamente, de cada uno de los censos correspondientes para cada año: INSTITUTo NACIONAL DE EsTADÍsTiCA y Geografía (INEGI), Censo Nacional de Impartición de Justicia Estatal, [en línea], disponible en: https://www. inegi.org.mx/programas/cnije/2013/ (consultado el 23 de julio de 2019).

6 Ídem.

7 Ídem.

8 Ídem.

9 Instituto Nacional de Estadística y Geografía (INEGI), Censo Nacional de Impartición de Justicia Estatal 2014, [en línea], disponible en: https://www.inegi.org.mx/programas/cnije/2014/default.html\#Tabulados (consultado el 25 julio de 2019)

${ }^{10}$ Sin tener en cuenta el 2013, año en que no se reportó ningún valor en las sentencias en materia de delitos sexuales en el estado de Guanajuato, excepto para el delito de violación (simple) e incesto. Esto equivale, no obstante, en números absolutos, a seis sentencias sobre un total de 289 procesados por delitos sexuales. Así se verificó hasta la revisión previa a la presentación de este artículo para su dictamen correspondiente. Los criterios de búsqueda empleados fueron los siguientes: “Sentenciados. Por: Entidad. Según: Tipo de delito”. Ídem.

${ }^{11}$ Instituto Nacional de Estadística y Geografía (INEGI), op. cit., nota 7.

${ }^{12}$ Lo anterior fue verificado hasta el momento previo al envío de este trabajo para su dictamen respectivo. Los criterios de búsqueda fueron los siguientes: "Sentenciados. Por: Entidad. Según: Tipo de delito". Instituto NacioNAl de Estadística y Geografía (INEGI), Censo Nacional de Impartición de Justicia Estatal 2018, [en línea], disponible en: https://www.inegi.org.mx/programas/cnije/2018/default.html\#Tabulados (consultado el 23 agosto de 2019). 
de ellos son cometidos por varones, así se constata en las sentenciadas emitidas por el poder judicial, particularmente en los casos de abuso sexual y violación. Con base en la información aquí recabada, y a manera de ejemplo de una problemática social de amplio alcance, puede sostenerse que el ejercicio de la violencia sexual en Guanajuato es, definitivamente, masculina.

\subsection{Hallazgos estadísticos}

Cabe hacer algunas observaciones en torno a los registros estadísticos que hasta ahora se han mostrado, mismos que pueden resumirse de la siguiente manera:

Violencias sexuales en Guanajuato 2010-2017

\begin{tabular}{|c|c|c|c|}
\hline Delitos & $\begin{array}{l}\% \text { hombres } \\
\text { Procesados }\end{array}$ & $\begin{array}{l}\% \text { hombres } \\
\text { Sentenciados }\end{array}$ & $\begin{array}{c}\text { \% sexo no } \\
\text { identificado } \\
\text { (únicamente año } \\
\text { 2013) }\end{array}$ \\
\hline Violencias sexuales ${ }^{*}$ & 87.68 & 99.16 & 83.73 \\
\hline Abuso sexual & 98.78 & 98.90 & 87.70 \\
\hline Violación simple & 96.84 & 99.37 & 75.53 \\
\hline
\end{tabular}

Fuente: elaboración propia con datos del INEGI, Censo Nacional de Impartición de Justicia Estatal, ediciones 2011 a 2018.

* Los años 2010-2012 se reportaron como delitos sexuales: abuso sexual, violación simple e incesto; el 2013, además de los anteriores: acoso sexual, hostigamiento sexual, violación equiparada y otros delitos sexuales; el 2014, además de los anteriores: estupro, "otros delitos sexuales sin realización de cópula" y "otros delitos sexuales con realización de cópula"; estos dos últimos delitos no se reportan el 2015 ni el 2016. Sin embargo, el 2017, además de los anteriores, se reportan los delitos de "violación equiparada a menores", "otro tipo de violación" y "violación no especificado".

En primer lugar, es destacable la sobrerrepresentación masculina en lo relativo a procesados y sentenciados, lo cual implica importantes proporciones de culpabilidad de los delitos sexuales cometidos en Guanajuato. Aun cuando constituyen tan sólo un ejemplo de dicha responsabilidad, los valores antes anotados son destacables dada la importante presencia de los hombres, de 9 a 1 respecto de las mujeres, tanto en la procuración como en la administración de justicia.

La segunda observación tiene que ver con el sexo de las personas procesadas. Cabe llamar la atención en el porcentaje de procesados reportado (87.68\%) por delitos sexuales en el estado de Guanajuato, ya que en el contexto nacional dichos valores se encuentran aproximadamente diez puntos porcentuales por encima de dicho valor, al menos así acontece en el período 2010-2017 ${ }^{13}$.

Esta situación tiene una explicación para el caso concreto y es conveniente comentarla. La página electrónica del Censo Nacional de Impartición de Justicia Estatal permite diversas opciones de búsqueda. En la edición del año 2014 (que ofrece datos del 2013), bajo diversos criterios de consulta, como son tipo de delito sexual, ámbito geográfico, personas procesadas y sexo;

13 En la República Mexicana, en lo relativo al conjunto de delitos sexuales, en el período 2010-2017, en promedio, los varones fueron procesados en un 97.19\%; y sentenciados en un 98.31\%. Es destacable también, como se señaló al principio de este trabajo, la presencia masculina si se toman como referente los delitos de violación simple y de abuso sexual. Instituto Nacional De Estadística y Geografía (INEGI), op. cit., nota 7. 
para el conjunto de delitos sexuales, en promedio, se arrojan los siguientes resultados: $16.26 \%$ de hombres procesados, ninguna mujer y, cuestión destacable, un $83.73 \%$ de personas con sexo no identificado ${ }^{14}$. Es decir, en lo relativo al conjunto de delitos sexuales, de cada diez, en (poco más de) ocho no se pudo identificar si las personas procesadas eran hombres o mujeres.

De esta forma, si sólo se tienen en cuenta los casos en que sí se identificó el sexo de la persona, en promedio, en el período 2010 a 2017 , los varones fueron procesados en un $98.15 \%{ }^{15}$. Valor que se diferencia en poco más de diez puntos porcentuales para el anotado en el apartado anterior, pero más próximo a la tendencia de este conjunto de delitos en el ámbito nacional y también para otros años del mismo estado de Guanajuato. Es destacable, tal como puede observarse, que valores elevados de una variable pueden impactar de forma importante en un período de tiempo más o menos prolongado. En el caso concreto, el sexo no identificado en altas proporciones, en un solo año, tuvo un impacto hasta de diez puntos porcentuales en el período de ocho años.

Cabe preguntarse, para terminar esta observación, qué ocurrió con los registros que reporta el INEGI y las autoridades guanajuatenses, especialmente en el año 2013 y 2017; tanto en lo que respecta al sexo de las personas como a los registros de los delitos de violación en particular. Las altas proporciones de personas con sexo identificado, de delitos no especificados, así como la falta de datos en algunos años y/o delitos específicos, sugieren importantes deficiencias en la forma de captar y reportar la información, e incluso, permiten poner en duda tales datos y, al menos en algunos aspectos, años y/o delitos, se podría poner en duda su validez.

Una tercera observación gira en torno a los delitos sobre los cuales da información el INEGI, ya que, en algunos años, al menos desde el 2010, los tipos penales reportados varían. Los años 2010-2012, por ejemplo, no se reportan datos de acoso sexual, hostigamiento sexual, violación equiparada ni otros delitos que atentan contra la libertad y seguridad sexual; pero, sí se reporta el delito de aprovechamiento sexual. En los años 2013-2017 ya no aparece en los registros este último delito, los demás sí son consignados. Sin embargo, resultan especialmente interesantes las modalidades del delito de violación que se reportan. El 2013-2017 se consigna la violación (simple) y la violación equiparada, el 2017, además de las anteriores, se reporta la violación equiparada en contra de menores e incapaces, así como también, otro tipo de violación y violación no especificado. Por su parte, el 2014, aun cuando no se consignan esta dos últimas modalidades de violación, se reportan otros delitos sexuales sin realización de cópula y otros delitos sexuales con realización de cópula ${ }^{16}$.

$\mathrm{Al}$ respecto, por una parte, es importante tener en cuenta que en la Norma Técnica de Delitos del Fuero Común ${ }^{17}$ para fines estadísticos no está considerada la clasificación de las modali-

\footnotetext{
${ }^{14}$ En este año, los delitos reportados fueron: Abuso sexual, acoso sexual, violación simple, violación equiparada, incesto y otros delitos sexuales. Cabe señalar que en lo relativo al reporte de personas cuyo sexo no fue identificado, al menos para el período de referencia, sólo acontece en el año 2013 y 2015, pero en este último caso, dicho valor fue mínimo. Para el caso del año 2013, ver: Instituto Nacional De Estadística y Geografía (INEGI), op. cit., nota 11; para el caso del año 2015, ver: Instituto Nacional De Estadística y Geografía (INEGI), Censo Nacional de Impartición de Justicia Estatal 2016, [en línea], disponible en: https://www.inegi.org.mx/programas/cnije/2016/default.html\#Tabulados (consultado el 23 julio de 2019).

15 Instituto Nacional De Estadística y Geografía (INEGI), op. cit., nota 7.

${ }^{16}$ Instituto Nacional De Estadística y Geografía (INEGI), op. cit., nota 7.

${ }^{17} \mathrm{El}$ año 2011 se emitió una norma técnica para la clasificación de delitos, esta es de carácter obligatorio y tiene el objetivo de perfeccionar el registro estadístico de los delitos del fuero común. Así mismo, esta norma fue modificada el año 2018, no obstante, se usa la de 2011 por ser la vigente al período de tiempo que se hace referencia en este estudio. Secretaria De Gobernación (2011), "Norma técnica para la clasificación nacional de delitos del fuero común para fines estadísticos”, Diario Oficial de la Federación, México, Secretaria de Gobernación, artículos 1, 2 y 8.
} 
dades de los ilícitos: otros delitos sexuales sin realización de cópula, otros delitos sexuales con realización de cópula, otro tipo de violación y violación no especificado. Por otro parte, lo más importante, es que estas conductas, al menos bajo tales nombres, no están tipificados en el Código Penal del estado de Guanajuato ${ }^{18}$. Además, cabe preguntarse diversas cuestiones: cuál sería la diferencia entre otro delito (sexual) con cópula y la violación; qué conducta constituye otro tipo de violación cuando se cuenta con diversos tipos penales relativos a este delito. También queda la duda, en cuanto a otros delitos sexuales sin realización de cópula, en qué medida estos podrían constituir abusos sexuales.

Una cuarta observación tiene que ver con la página electrónica del Censo Nacional de Impartición de Justicia Estatal, en particular, en lo relativo a las actualizaciones de la información que en ella se reporta. A lo largo del desarrollo de esta investigación pudieron observarse diversas actualizaciones sin que se realizaran los avisos respectivos de forma oportuna ${ }^{19}$. Así aconteció, por ejemplo, de manera específica en lo relativo a los datos de violencias sexuales, del año 2016, en el estado de Guanajuato, así como también en algunos casos de sentencias ${ }^{20}$.

Es de suma importancia la actualización de los datos que reportan tanto las autoridades del sistema de justicia, así como del INEGI, no obstante, tiene la misma importancia informar de manera oportuna a los usuarios de los registros estadísticos. Al respecto, cabe preguntarse ¿con qué frecuencia se realizan tales actualizaciones?, ¿de qué manera informan las autoridades los cambios que realizan?, sobre todo, ¿en qué clase de errores o descuidos se basan las omisiones que posteriormente requieren actualizaciones?

Los datos comentados, además de la culpabilidad de los varones, sugieren necesidades apremiantes en sentido estadístico, en las formas en que se capta la información así como la manera en que esta es reportada, particularmente, como se ha observado en este capítulo, en cuanto al sexo de las personas. En este sentido, se evidencia y confirma, como señala Kala, la escasa tradición estadística en las ciencias penales mexicanas ${ }^{21}$.

\section{El género masculino y la tradición criminológica}

En este apartado se analiza la forma en que los varones y el género masculino han sido concebidos en las explicaciones de tres grandes perspectivas criminológicas del siglo XX, el positivismo, la perspectiva sociológica, tanto en su vertiente de la Escuela de Chicago como de las teorías sub-culturales; y, finalmente, una óptica que puede denominarse de la diferencia sexual. Se estudia la forma en que estas han tenido en cuenta la sexualidad masculina en sus explicaciones en torno a los delitos que atentan contra la libertad sexual.

\footnotetext{
${ }^{18}$ Código Penal del Estado De Guanajuato (2019), op. cit., nota 3.

19 Al respecto, véase supra no. 5; también,Instituto Nacional De Estadística y Geografía (INEGI), Censo Nacional de Impartición de Justicia Estatal 2019, [en línea], disponible en: https:/www.inegi.org.mx/programas/ cnije/2019/ (consultado el 17 de agosto de 2019).

${ }^{20}$ A lo largo del desarrollo de esta investigación, el mes de abril de 2019 se hicieron diversas consultas en el Censo Estatal de Impartición de Justicia Estatal 2017 (que reporta datos de 2016) y no se reportaban datos relativos a las violencias sexuales en el estado de Guanajuato para 2016. No obstante, el mes de julio de 2019 ya se reportaban dichos datos en la página web de dicho censo. Además de ello, para el mismo año, se llevaron a cabo actualizaciones en torno a las sentencias, por ejemplo, al realizar algunas consultas en abril de 2019, pudo observarse que se reportaron 25,792 sentencias en todo el país; en julio del mismo año, se reportaron un total de 29,086. INSTITUTO Nacional De Estadística y Geografía (INEGI), Censo Nacional de Impartición de Justicia Estatal 2017, [en línea], disponible en: https://www.inegi.org.mx/programas/cnije/2017/ (consultado el 25 julio de 2019).

${ }^{21}$ Kala, Julio César (2003), Fenomenología de la Delincuencia. Ciudades Seguras IV, México, FCE, p. 8.
} 


\subsection{El positivismo: la esencialización de la sexualidad}

La etapa científica de la criminología o positivismo está representada por autores como Lombroso, Ferri o Garófalo, quienes desarrollaron una serie de postulados que se caracterizan por su androcentrismo y su sexismo ${ }^{22}$. En este sentido son ilustrativos los atributos asignados al varón delincuente: gran resistencia física, e incluso, invulnerabilidad ${ }^{23}$; rasgos fisonómicos determinados $^{24}$ y definidos en términos de fealdad ${ }^{25}$; una gran potencia sexual ${ }^{26}$, o bien, considerar que el hombre se veía estimulado al delito dado el orgullo, la venganza, la avaricia y/o el sentido de los placeres, que se desencadena indómito en las pasiones del erotismo, del juego, de la gula y de la orgia ${ }^{27}$.

La sexualidad del varón delincuente, por su parte, es asumida acríticamente y se enaltece la virilidad, entendida esta como aquella cualidad indisociable, por lo menos tácitamente, de la virilidad física, a través especialmente de las demostraciones de fuerza sexual 3/4desfloración de la novia, abundante progenie masculina, etc. $3 / 4$ que se esperan del hombre que es verdaderamente hombre ${ }^{28}$. Este aspecto queda reflejado en la idea del ser activo sexualmente, ya que, además de la heterosexualidad, se suponía que el varón tenía preferencia por un papel activo y se revela contra la pasividad, ya que ésta última es principalmente femenina ${ }^{29}$.

La noción activo-masculino/pasivo-femenino tuvo una importante presencia en la concepción positivista de la sexualidad y además se vinculó a la violencia ${ }^{30}$. Esta dualidad está presente en Lombroso ${ }^{31}$, por ejemplo, cuando analiza lo que denomina usos y costumbres de los pueblos primitivos, al respecto, sostiene que el órgano viril, que indica fuerza, sirve precisamente para indicar [...] el valor de los pueblos que le opusieron resistencia; y, al contrario, la vulva representa la debilidad de los pueblos fácilmente subyugados ${ }^{32}$.

La etapa científica de la criminología favoreció la construcción del delincuente como un adulto, varón e hipermasculinizado ${ }^{33}$. Su sexualidad sencillamente no es cuestionada y, lo más importante, se vinculó a la fuerza y la agresividad; es una condición que acaba por ser esencializada. Esta concepción, puede decirse, es una idea virilizada de la sexualidad de los varones:

\footnotetext{
${ }^{22}$ Smart, Carol (1977), Women, crime and criminology: a feminist critique, London, Routledge \& Kegan Paul; SMART, Carol (1995), Law, crime and sexuality. Essays in feminism, London, SAGE.

${ }^{23}$ Lombroso, Cesare (1920), Los criminales, Barcelona, Editorial Atlante, pp. 37-39.

${ }^{24}$ Entre otros, cabe destacar la asimetría del cráneo y de la cara, mandíbulas voluminosas, pómulos salientes, determinada forma de las orejas y del mentón, nariz torcida o chata, frente hundida, abundancia de cabello o ausencia de barba. Lombroso, Cesare (1975), Lombroso y la Escuela Positiva Italiana, Madrid, CSdeIC, pp. 257-258.

${ }^{25}$ GARófAlo, Raffaele (2005), La criminología. Estudio sobre el delito y la teoría de la represión, Argentina, BdeF, p. 69.

${ }^{26}$ Von KRAFFt-EBing, Richard (2009), "Pathological sexuality", The origins of criminology, a reader, edited by Nicole Rafter, Great Britain, Routledge, p. 67; Lombroso, Cesare (1920), op. cit., nota 25.

27 Ferri, Enrico (2005), Sociología criminal, Navarra, España, Analecta ediciones, p. 61.

${ }^{28}$ Bourdieu, Pierre (200o), La dominación masculina, Barcelona, Anagrama, p. 25.

${ }^{29}$ Freud, Sigmund (2006), Tres ensayos sobre la sexualidad, Madrid, Alianza, pp. 90-91. Es destacable que algunas características tradicionalmente consideradas como inherentes de la sexualidad del varón se han sustentado en planteamientos desarrollados por Freud y han tenido un impacto importante en la criminología. En este sentido, ver: TAYlor, Ian, et. al., (1990), La nueva criminología. Contribución a una teoría social de la conducta desviada, Argentina, Amorrortu.

30 Von Krafft-Ebing, Richard (2009), op. cit., nota 28, p. 67.

${ }^{31}$ Lombroso, Cesare (1975), op. cit., nota 26, p. 265; Lombroso And Ferrero (2004), Criminal woman, the prostitute and the normal woman, trad., Nicole Hahn Rafter and Maty Gibson, Duke University Press, Durham.

${ }^{32}$ Lombroso, Cesare (1975), op. cit., nota 26, p. 265

${ }^{33}$ Rodríguez LunA, Ricardo (2018). "La exaltación de lo viril en el positivismo criminológico: la masculinidad en el delincuente del siglo XX”, Alegatos, UAM-Azcapotzalco, núm. 99, mayo agosto, pp. 309-324.
} 
heterosexual, activa, agresiva o de gran potencia. Este aspecto queda reflejado, incluso, cuando Lombroso explica la delincuencia de las mujeres ${ }^{34}$, ya que considera esta criminalidad como resultado de la sexualidad y la acaba por identificar con la prostitución, misma que, además, representa [...] el equivalente de la criminalidad masculina ${ }^{35}$.

Para finalizar este apartado, puede sostenerse que el positivismo criminológico, al asumir una concepción esencializada de la sexualidad masculina, concibió las violencias sexuales de los varones de manera acrítica y como un aspecto que formaba parte de la naturaleza de ser hombre. Fue obviada la condición de género masculino, esta se dio por sentada, fue concebida como natural, inmutable e incuestionable ${ }^{36}$. A su vez, la sexualidad del varón se enalteció, se asoció a la virilidad, al ser activo, la potencia, la heterosexualidad y, también, a la agresividad.

\subsection{La óptica sociológica: el afianzamiento de los roles socio-sexuales}

Los postulados del positivismo fueron severamente cuestionados a lo largo del siglo XX, en un primer momento, de ello se encargó la Escuela de Chicago ${ }^{37}$, posteriormente, en el segundo tercio de dicho siglo, la crítica estuvo a cargo de las teorías subculturales del delito. En cuanto a los teóricos de Chicago, a pesar de su postura crítica, su perspectiva se basó en un punto de vista evolucionista ${ }^{38}$ de la organización social, en consecuencia, adoptaron una noción de división sexual del trabajo en donde el individuo tenía funciones determinadas, entre otras: la mujer cuida $\mathrm{y}$ alimenta, el varón provee y protege ${ }^{39}$.

Esta concepción favoreció que en la criminología se consolidara la idea del varón cabeza de familia y padre ausente ${ }^{40}$; además, los hombres y lo masculino estuvieron representados a través de imágenes determinadas: violencia, bebida, negligencia económica, incumplimiento de pensión, conducta desordenada, adulterio, no expresión de sentimientos ${ }^{41} \mathrm{y}$ activo en aspectos como la lucha, la defensa o las relaciones sexuales ${ }^{42}$. Interesa, no obstante, situar algunos de es-

\footnotetext{
34 Lombroso And Ferrero (2004), op. cit., nota 33, p. 171.

${ }^{35}$ Lombroso, Cesare (1975), op. cit., nota 26, p. 233.

36 Smart, Carol (1977), op. cit., nota 24; Connell, Raewyn W. (2003), Masculinidades, México, PUEGUNAM.

37 Se conoce con este nombre al corpus de textos sociológicos producidos principalmente en el periodo de entreguerras por el Departamento de Sociología de la Universidad de Chicago, "y cuyas consecuencias teóricas pueden reconocerse en muchas de las corrientes que nacen de la aludida Escuela de Chicago". BERGALLI, Roberto (1983), "Perspectivas sociológicas: desarrollos ulteriores", El pensamiento criminológico I. Un análisis crítico, Barcelona, Península, p.113.

${ }^{38}$ En general, puede hablarse de una visión organicista-ecológica que favoreció una concepción de la división del trabajo determinada por condiciones comunes a los mamíferos, como es el suministro de alimentos, las adaptaciones y los cambios morfológicos en la evolución de las especies o la sobrevivencia del grupo. Un elemento especialmente destacable en esta forma de concebir la organización social, de acuerdo con Chodorow, es el ejercicio de la maternidad, función necesaria para perpetuar la especie, que debe ser llevada a cabo por las mujeres y que constituye "uno de los pocos elementos universales y permanentes de la división sexual el trabajo". CHODOROW, Nancy (1984), El ejercicio de la maternidad, España, Gedisa, p. 13; PARK, Robert (1999), La ciudad y otros ensayos de ecología urbana, España, Serbal, p. 131.

39 Thomas, William I., (1906), “The adventitious character of woman”, American Journal of Sociology, núm. 1, p. 34.

40 Thomas William I. y Znaniecki, Forian (2004), El campesino polaco en Europa y en América, Madrid, Centro de Investigaciones Sociológicas / Agencia Estatal Boletín del Estado, p. 172.

41 Ídem.

42 Thом as, William I., (1899), “Sex in primitive morality”, American Journal of Sociology, núm. 4, p. 775.
} 
tos elementos en el marco de las explicaciones del entorno urbano desarrolladas por los teóricos de Chicago.

Consideraron que la ciudad poseía una organización social y moral propiamente urbanas, misma que se caracterizaba por relaciones e interacciones sociales ${ }^{43}$ inexistentes hasta entonces. Estas fueron, sin embargo, definidas en términos y cualidades masculinas: racionalidad, impersonalidad, interés o dinero, además, y cuestión importante, formaban parte de la moral vigente de las ciudades. A su vez, la moral divergente se asoció a atributos femeninos: lo irreflexivo, lo innato o natural ${ }^{44}$. Es destacable que las regiones en donde predominan códigos morales divergentes eran consideradas como áreas gérmenes del delito y en ellas los individuos eran dominados por sus pasiones ${ }^{45}$.

Los teóricos de Chicago reprodujeron diversos estereotipos de género, en particular, la concepción de sentimientos y pasiones como algo irracional y negativo que contribuían a la desorganización social ${ }^{46}$. Además, realizan asignaciones y atribuyen roles a hombres y mujeres que no cuestionan ni problematizan la concepción del hombre cabeza de familia ni de paternidad ausente. Tampoco se preguntan por qué la desorganización social y moral ${ }^{47}$ ejercía una mayor influencia en los hombres que en las mujeres, o bien, porqué estas se adaptaban mejor a los cambios que suponía la ciudad.

De esta forma, en relación con la problemática planteada en este trabajo, puede sostenerse que la Escuela de Chicago no responde por qué la desorganización impactaba en mayor medida en el varón y, en consecuencia, lo lleva a cometer muchos más delitos que las mujeres. Consideraron que el varón, si se dejaba llevar por sus sentimientos y pasiones innatas, entonces divergía del orden establecido y se constituía en parte de la desorganización. El delincuente varón sería un desorganizado, es decir, aquel individuo alejado de su papel hombre cabeza de familia, pero no así, de las asignaciones sociales que determina la moral masculina vigente, además, cuando se veía arrastrado por sus más profundas e innatas pasiones 3/4irracionales e irreflexivas; femeninas $3 / 4$ estas lo conducirían a cometer un delito.

En términos de sexualidad masculina, la Escuela de Chicago, la asocia a ideas como infidelidad, no expresión de sentimientos y asume la noción de actividad/pasividad. Por su parte, en

${ }^{43}$ De acuerdo con Park, estas se denominaron secundarias, en contra posición a las relaciones "cara a cara", y su importancia derivó de constituir sentimientos, interacciones y relaciones propiamente urbanas; además de contar con su propio orden, regiones y climas morales. No obstante, a su vez, existían diversas formas de desorganización social, es decir, "una reducción de la influencia de las reglas sociales de conducta existentes entre los miembros individuales del grupo". Es decir, en el contexto de la emergencia de los centros urbanos - que dejan atrás a la comunidad-, la adaptación del individuo a los nuevos hábitos y costumbres puede manifestarse como una "simple ruptura de una regla particular [...] hasta el desmoronamiento general de todas las instituciones del grupo”. Thomas William I. y ZnANiecki, Forian (2004), op. cit., nota 42, p. 305; ver también PARK, Robert (1999), op. cit., nota 40.

${ }^{44}$ Сово, Rosa (1995), Fundamentos del patriarcado moderno. Jean Jaques Rousseau, Madrid, Cátedra, p. 22. Ver también: NASH, Mary (2004), Mujeres en el mundo. Historia, retos y movimientos, Madrid, Alianza, pp. 34-50; SeIdler, Victor (2000), La sinrazón masculina. Masculinidad y teoría social, México, Paidós.

${ }^{45}$ PARK, Robert (1999), op. cit., nota 40, p. 83; Thomas William I. y Znaniecki, Forian (2004), op. cit., nota 42.

${ }^{46} \mathrm{Se}$ favorece así, de acuerdo con Taylor, la reproducción de un vínculo entre pasiones-sentimientos-delito, en donde el binomio sentimiento-pasión se relaciona con lo irreflexivo, instintivo, natural; todo ello, históricamente femenino. TAYLOR, Ian, et. al., (1990), op. cit., nota 31, p. 22.

${ }^{47}$ Esta se definió como "una reducción de la influencia de las reglas sociales de conducta existentes entre los miembros individuales del grupo". Es decir, en el contexto de la emergencia de los centros urbanos - que dejan atrás a la comunidad-, la adaptación del individuo a los nuevos hábitos y costumbres puede manifestarse como una "simple ruptura de una regla particular [...] hasta el desmoronamiento general de todas las instituciones del grupo". Thomas William I. y ZnANiecKi, Forian (2004), op. cit., nota 42, p. 305. 
el marco de la ciudad, plantea la existencia de una organización social y moral urbana que, por un lado, es racional, masculina; por otro, la desorganización es irreflexiva, atrae y despierta lo natural e innato del individuo, es irracional y femenina. De esta forma, tampoco cuestionan lo masculino ni cómo esto se asocia al delito, más bien, contribuyen a la reproducción de lo masculino-viril como algo intrínseco de la naturaleza del varón.

En el segundo tercio del siglo XX, por otra parte, se consolidó la influencia sociológica en la criminología, en buena medida gracias a Robert Merton. Aspecto que se vio reflejado en las teorías de la tensión y la frustración social, cuyos principales representantes son afines a las teorías subculturales del delito, entre otros, A. K. Cohen ${ }^{48}$ y R. Cloward \& L. Ohlin ${ }^{49}$.

Estas teorías asumieron la noción de rol sexual que se consolidó en este período. Los roles fueron considerados como unidades del sistema social ${ }^{50}$, es decir, no eran atributos del propio sujeto y para evitar disfuncionalidades en dicho sistema, se consideró de la mayor importancia que el individuo se comportara conforme a diversos criterios normativos $3 / 4$ culturales $3 / 4$ que establecían cómo debería de ser su conducta ${ }^{51}$. Esta idea adquiere capital importancia cuando se plantea en términos de la diferencia sexual, ya que se requiere orientar la actuación de las personas hacia determinados criterios normativos según su sexo. De esta forma, cobra trascendencia el actuar del individuo en sus relaciones con el otro sexo, así como lo que se espera de determinada persona, en tanto mujer u hombre, y conforme a las exigencias de roles en el contexto de un conjunto de valores compartidos. Son ilustrativos los elementos que, de acuerdo con Parsons, estructuran el rol sexual masculino:

Mayor prestigio y responsabilidad; gira en torno a la heterosexualidad y la homosexualidad es planteada como un problema, es decir, se supone que los roles tendrían un carácter complementario. El hombre ejerce una paternidad habitualmente ausente, aunque posee una gran importancia simbólica en términos de identificación del niño con el rol masculino ${ }^{52}$; los niños rehúyen las expresiones de sentimientos tiernos; han de ser 'duros'53, además, cuando estos entran al período de latencia, su comportamiento tiende a caracterizarse por una especie de masculinidad compulsiva ${ }^{54}$.

En el ámbito criminológico, la idea de rol sexual es asumida por A. K. Cohen ${ }^{55}$ y R. Cloward \& L. Ohlin ${ }^{56}$. Sostienen la existencia de una cultura dominante o un sistema de valores compartidos, al hacerlo, asumen diversos estereotipos de género y otorgan un importante papel a la masculinidad y a la feminidad. Sin embargo, en relación con el problema de estudio planteado en esta investigación, de acuerdo con M. Chesney-Lind \& R. G. Shelden ${ }^{57}$, Cohen plantea que el "delincuente es el macho dominante" y que su esencia masculina se afirma al ser condenado moralmente por los otros. Por su parte, las explicaciones de Cloward \& Ohlin hacen referencia

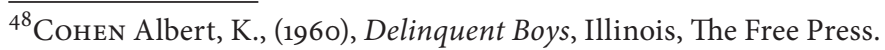

${ }^{49}$ Cloward, Richard \& Ohlin, Lloyd (1967), Delinquency and opportunity. A theory of delinquent gangs, Canadá, Collier-Macmillan.

50 PArsons, Talcott (1984), El sistema social, Madrid, Alianza, p. 34-44.

${ }^{51}$ Ibidem, p. 44.

52 Ibidem, p. 231 y ss.

${ }^{53}$ Parsons, Talcott (1972), "La estructura social de la familia”, La familia. Fromm, Horkheimer, Parsons, Barcelona, Península, p. 47.

54 Ídem.

55 Cohen Albert, K., (1960), op. cit., nota 50.

${ }^{56}$ ClOWARD, Richard \& OHLin, Lloyd (1967), op. cit., nota 51.

57 Chesney-Lind, Meda y Shelden, Randall G., (2004), Girls, delinquency and juvenile justice, USA, Wadsworth, pp. 113-114.
} 
a la protesta masculina o masculinidad compulsiva, misma que los conduce a protestar contra la feminidad. Es decir, dado que el padre está generalmente ausente del hogar, los niños tienen problemas para formarse una imagen masculina. Aunado a ello, especialmente en la adolescencia, los jóvenes se dan cuenta de la existencia de expectativas culturales que les indican el deber de comportarse como hombres. Inmersos en un mundo en donde la madre ha sido el objeto de identificación y con dificultades propias relativas a su identidad, los jóvenes experimentan una gran tensión y protestan contra la feminidad ${ }^{58}$. Al respecto, las explicaciones de la tensión y la frustración social se caracterizan por ser acríticas referente a los roles de género tradicionales ${ }^{59}$.

Para finalizar este apartado, puede decirse que las perspectivas sociológicas sentaron las bases de una fuerte crítica a la tradición positivista que les antecedió y favorecieron el desarrollo de nuevas explicaciones en torno al delito. Esta situación permitió que se restara cierta hipermasculinización al delincuente, pero, a su vez, favoreció una interpretación funcionalista y una fuerte orientación a la noción de roles sociales y sexuales. Al hombre se le concibió preeminentemente como jefe o cabeza de familia, proveedor y protector. Así, los hombres, lo masculino y el delito estuvieron vinculados de forma importante con diversos roles que en función del género se suponía debían cumplir los varones.

\subsection{La óptica de género: el desarrollo de una perspectiva de lo masculino}

El feminismo y los estudios de género han sido actores principales de diversos cambios epistemológicos que han tenido lugar en la criminología, principalmente desde los años setenta y hasta la actualidad ${ }^{60}$. Uno de los más importantes, acontecido principalmente desde la década de los años noventa del siglo pasado, ha consistido en señalar la necesidad de desarrollar acciones orientadas a los hombres, fomentar su participación en la prevención, eliminación y lucha contra el sexismo, así como todas las formas de discriminación y violencia contra las mujeres ${ }^{61}$. Este cambio ha favorecido, dicho en forma resumida, que los varones sean tenidos en cuenta como sujetos con determinadas y específicas condiciones en función de la construcción del ser hombres y los significados que ello adquiere en sus vidas cotidianas.

El desarrollo de esta visión ha favorecido la incorporación de la noción de masculinidades al ámbito de las ciencias penales; concepción que ha sido definida como un lugar en las relaciones de género, en las prácticas a través de las cuales los hombres y las mujeres ocupan ese espacio en el género, y en los efectos de dichas prácticas en la experiencia corporal, la personalidad y la cul-

\footnotetext{
${ }^{58}$ Cloward, Richard \& Ohlin, Lloyd (1967), op. cit., nota 51, p. 63.

59 Chesney-Lind, Meda y Shelden, Randall G., (2004), op. cit., nota 59.

60 Entre otras cuestiones, cabe destacar que se ha caracterizado por la crítica y la visibilización de posturas que vinculan de forma sexista el crimen y la diferencia sexual. Por un lado, ha mostrado la existencia de una concepción dominante en torno a las mujeres, su "naturaleza" y la forma en que ello se relacionaba con la delincuencia. Por otro lado, ha subrayado que se habían desarrollado explicaciones de la delincuencia de los hombres y estas se había hecho extensivas a las mujeres. Chesney-Lind, Meda y Shelden, Randall G., (2004), op. cit., nota 57; Maqueda, María Luisa (2014), Razones y sinrazones para una criminología feminista, Madrid, Instituto Andaluz Interuniversitario de Criminología/ Dykinson; SMART, Carol (1977) y (1995), op. cit., nota 24.

${ }^{61}$ Unión Europea (2002), Protección de las mujeres contra la violencia. Recomendación Rec(2002) 5; Unión Europea (2006), Dictamen. La violencia doméstica contra las mujeres. Núm. 2006/C 110/15; Unión EUROPEA, (2011), Pacto europeo por la igualdad de género (2011-2020). Bruselas, Unión Europea. Doc. 2011/C 155/O2; BARKER, Gary, et. al., (2011), Políticas de equidad de género inclusivas de los hombres: reflexiones a partir de la encuesta IMAGES y una revisión de políticas en Brasil, Chile y México, Río de Janeiro, Promundo; Flood, Michael (2011), "Involving men in efforts to end violence against women", Men and masculinities, 14, pp. 358-377; OrGANIZACión De Las Naciones Unidas (ONU) / Comisión de la Condición Jurídica y Social de la Mujer, (2013), Informe sobre el $57^{\circ}$ periodo de sesiones, Doc. E/2013/27E/CN.6/2013/11, Nueva York, ONU.
} 
tura $^{62}$. Esta definición no ha estado ausente de polémicas ni polisemias, pero existe un amplio consenso en considerar lo masculino como una construcción social relacionada con contextos históricos y socioculturales determinados.

La noción de masculinidad se ha incorporado poco a poco en los estudios criminológicos, al hacerlo, se han destacado los procesos de socialización que dotan a los varones de determinados modelos de lo que significa ser hombre y de aquello que constituye la masculinidad ${ }^{63}$. Éstas, es importante destacar, se desarrollan en un contexto social patriarcal, lo cual se traduce en importantes vínculos con el poder y el control ${ }^{64}$, situación que tiene diversos costos para los varones, uno de los principales es la constante relación con la violencia ${ }^{65}$. De hecho, se ha sostenido que llegar a ser hombre implica experiencias y procesos de socialización que favorecen los vínculos con el delito ${ }^{66}$ y con la violencia, ya que esta ha sido parte del significado de la masculinidad, parte de la forma en que los varones han medido, demostrado y probado su identidad ${ }^{67}$. Aunque, cabe subrayarlo, no todos los hombres ni las masculinidades son violentas.

Desde esta perspectiva, en relación con el problema de estudio de este trabajo, puede sostenerse que la sexualidad masculina ha sido construida bajo un modelo androcéntrico ${ }^{68}$ carac- $^{-}$ terizado porque la relación sexual constituye un acto de dominación ${ }^{69}$. Esto se traduce en una concepción de sexualidad conformada por elementos que han sido tradicionalmente enaltecidos, entre otros: la virilidad, la penetración, el dualismo activo-masculino/pasivo-femenino, el deber ser sexual, la fuerza y la violencia.

La virilidad dota de particulares connotaciones a hombres, a mujeres y las relaciones sociosexuales entre ambos, ya que, como se indicó en el apartado previo, permanece indisociable a la idea de virilidad física y a las demostraciones de fuerza sexual ${ }^{70}$. De igual forma, en el imaginario masculino, es especialmente destacable la idea de penetración, misma que constituye una de las afirmaciones de la libido dominandi ${ }^{71}$.

Penetración y dominación forman parte importante de la concepción androcéntrica de la sexualidad, ambas ideas quedan bien reflejadas en diversos dualismos que ilustran el marco in-

\footnotetext{
${ }^{62}$ Connell, Raewyn W. (2003), op. cit., nota 38, p.109.

${ }^{63}$ Bourdieu, Pierre (2000), op. cit., nota 30.

${ }^{6}$ Ídem. Bourdieu, Pierre (200o), op. cit., nota 30.

${ }^{65}$ Green, Judith (1997), "Risk and the construction of social identity: children's talk about accidents", Sociology of Health and Illness, no 4, vol. 19, pp. 457-479; KAUfmAN, Michael (1995), Los hombres, el feminismo y las experiencias contradictorias del poder entre hombres [en línea], disponible en: www.michaelkaufman.com (consultado el 7 de enero de 2019); KeIJzer Benno (1997), "El varón como factor de riesgo: masculinidad, salud mental y salud reproductiva”, Esperanza Tuñon (coord.), Género y Salud en el Sureste de México, México, El Colegio de la Frontera Sur / Universidad Juárez Autónoma de Tabasco; Kimmel, Michae., (1997), "Homofobia, temor, vergüenza y silencio en la identidad masculina", T. Váldes y J. Olavarría (eds.), Masculinidad/es, poder y crisis, Chile, Isis Internacional/Facultad Latinoamericana de Ciencias Sociales, pp. 49-62; Olavarría, José, (2006), "Hombres e identidad de género: algunos elementos sobre los recursos de poder y violencia masculina”, G. Careaga y S.Cruz (eds.), Debates sobre masculinidades, México, Programa Universitario de Estudios de Género-Universidad Nacional Autónoma de México; Bourdieu, Pierre (2000), op. cit., nota 30.

66 Stanko, Elizabeth (2009), "Es pot reduir la por de la delinqüència que tenen les dones", Revista Catalana de Seguretat Pública, no. 20, p. 36.

${ }^{67}$ Kimmel, Michael, (1997), op. cit., nota 67; Kimmel, Michael (2001), "Masculinidades globales: restauración y resistencia”, C. Sánchez y J. C. Hidalgo (eds), Masculino Plural: construcciones de la masculinidad, Lleida, Universitat de Lleida.

${ }^{68}$ Osborne, Raquel (2009), Apuntes sobre violencia de género, Barcelona, Ediciones Bellatera, pp. 70 y ss.

${ }^{69}$ Bourdieu, Pierre (2000), op. cit., nota 30; Osborne, Raquel (2009), op. cit., nota 70.

70 Bourdieu, Pierre (2000), op. cit., nota 30, p. 24.

${ }^{71}$ Ibidem, p. 35 .
} 
terpretativo del cuerpo y de las relaciones entre hombres y mujeres, por ejemplo, activo-pasivo, arriba-abajo, fuerte-débil, dominio-sometimiento, etc. Todos estos inmersos en el conjunto de comportamientos, interacciones y relaciones eróticas entre los sexos ${ }^{72}$, no obstante, los primeros términos de estas parejas dicotómicas están relacionados con lo masculino y supraordenados a los segundos términos, relacionados con lo femenino ${ }^{73}$. Esta concepción, basada en la jerarquía, pasó a conformar un deber ser sexual y una parte constitutiva de la masculinidad, ya que hombres y mujeres pasaron a ser vistos como dos variantes, superior e inferior, de la misma fisiología ${ }^{74}$.

Este último planteamiento es el que mejor responde al problema de estudio aquí analizado, ya que muestra, a la vez que cuestiona, el actual significado de lo masculino, de la sexualidad de los varones y sus vínculos con la agresividad y las violencias. Este sentido se evidencia con la sobrerrepresentación masculina de la responsabilidad de las violencias sexuales mostradas en el apartado previo, así como también la necesidad de intervenciones específicas en el ámbito de la sexualidad de los varones, de las representaciones y significados de sus relaciones socio sexuales, sea con personas o no del mismo sexo.

\section{Comentario final}

Los delitos analizados en esta investigación permiten sostener que los hombres están sobrerrepresentados en las estadísticas de delitos sexuales, sea que se trate de procesados o sentenciados. Así lo ilustran los datos del estado de Guanajuato analizados. Dicho en otras palabras, tal como muestran los registros estudiados, los varones fueron responsables de las violencias sexuales casi de manera exclusiva: la (des)proporción de su culpabilidad es incluso mayor de 9 hombres por 1 mujer, aspecto que se refleja en las sentencias judiciales en la materia. Todo ello se confirma especialmente en algunos delitos, como es el caso del abuso sexual o la violación, al menos, así aconteció en el periodo de tiempo aquí analizado.

Esta problemática en sí misma importante implica aspectos de diversa índole que no deben soslayarse. A lo largo de esta investigación emergió el caso de algunas deficiencias en los registros estadísticos del INEGI y/o de las autoridades penales de Guanajuato. Los datos empleados, aunque sólo se usaron para ejemplificar una situación, particularmente los del Censo Nacional de Impartición de Justicia Estatal, permiten observar inconsistentes e incluso en algunos casos es cuestionable su validez. Este fue el caso, de manera particular, de la variable sexo, ya que se detectaron importantes proporciones de personas cuyo sexo fue reportado como no identificado. Esta situación es especialmente destacable cuando se realizan búsquedas específicas que requieren el cruce de variables diversas, por ejemplo, edad, víctimas, sexo, un delito en particular, relación entre víctima o el sujeto activo del delito, los índices de responsabilidad y de victimización, si hay o no empleo de armas en la comisión de los delitos, o bien, los espacios en los que acontecen tales conductas.

Por otra parte, desde la perspectiva adoptada en este estudio, puede sostenerse que, al menos en parte, el grado de la responsabilidad masculina de las violencias sexuales responde a la

\footnotetext{
${ }^{72}$ Rubin, Gayle (1989), "Rlefexionando sobre el sexo: notas para una teoría radical de la sexualidad", en Carole S. Vance (comp.), Placer y Peligro. Explorando la sexualidad femenina, Madrid, Hablan las mujeres; GidDENs, Anthony (1995), La transformación de la intimidad. Sexualidad, amor y erotismo en las sociedades modernas, Madrid, Cátedra; Bourdieu, Pierre (2000), op. cit., nota 30.

73 Piтch, Tamar (2003), Un derecho para dos. La construcción jurídica de género, sexo y sexualidad, Madrid, Trotta, p. 253.

74 Bourdieu, Pierre (200o), op. cit., nota 3o, p. 28 (el subrayado es mío). 
forma en que se han construido socialmente los significados en torno a las masculinidades, en particular en lo relativo a la sexualidad de los varones y la forma en que esta es ejercida en las relaciones socio-sexuales con otras personas. Son especialmente destacables los vínculos entre agresividad, violencia, sexualidad y masculinidad. En este sentido, cabe señalar la necesidad de intervenciones públicas en la materia, así mismo, cabe cuestionar las estrategias y acciones públicas que se toman con base en estadísticas que muestran deficiencias como las encontradas a lo largo de esta investigación.

\section{Bibliografía}

BARKer, Gary et. al. (2011), Políticas de equidad de género inclusivas de los hombres: reflexiones a partir de la encuesta images y una revisión de políticas en Brasil, Chile y México, Río de Janeiro, Promundo.

Bergalli, Roberto (1983), "Perspectivas sociológicas: desarrollos ulteriores”, El pensamiento criminológico I. Un análisis crítico, Barcelona, Peninsula, pp. 109-132.

Bourdieu, Pierre (2000), La dominación masculina, Barcelona, Anagrama.

CHodorow, Nancy (1984), El ejercicio de la maternidad, España, Gedisa.

Chesney-Lind, Meda y Shelden, Randall G. (2004), Girls, delinquency and juvenile justice, USA, Wadsworth.

Cloward, Richard \& Ohlin, Lloyd (1967), Delinquency and opportunity. A theory of delinquent gangs, Canada, Collier-Macmillan.

Сово, Rosa (1995), Fundamentos del patriarcado moderno. Jean Jaques Rousseau, Madrid, Cátedra.

Código Penal del Estado de Guanajuato (2019), [en línea], disponible en: https://portal. pgjguanajuato.gob.mx/PortalWebEstatal/Archivo/normateca/2.pdf.

Cohen, Albert K. (1960), Delinquent Boys, Illinois, The Free Press.

Connell, Raewyn W. (2003), Masculinidades, México, PUEG-UNAM.

FERri, Enrico (2005), Sociología criminal, Navarra, España, Analecta ediciones.

FLooD, Michael (2011), "Involving men in efforts to end violence against women", Men and masculinities, 14, pp. 358-377.

Freud, Sigmund (2006), Tres ensayos sobre la sexualidad, Madrid, Alianza.

Garófalo, Raffaele (2005), La criminología. Estudio sobre el delito y la teoría de la represión, Argentina, BdeF.

Giddens, Anthony (1995), La transformación de la intimidad. Sexualidad, amor y erotismo en las sociedades modernas, Madrid, Cátedra. 
GreEN, Judith (1997), "Risk and the construction of social identity: children's talk about accidents", Sociology of Health and Illness, no 4, vol. 19, pp. 457-479.

Instituto Nacional de Estadística y Geografía (INEGI), www.inegi.com.mx

[en línea], disponible en: https://www.inegi.org.mx/programas/judicialespenal/default.html\#Tabulados

Censo Nacional de Impartición de Justicia Estatal 2012, [en línea], disponible en: https://www.inegi.org.mx/programas/cnije/2012/default.html\#Tabulados

Censo Nacional de Impartición de Justicia Estatal 2013, [en línea], disponible en: https:// www.inegi.org.mx/programas/cnije/2013/

Censo Nacional de Impartición de Justicia Estatal 2014, [en línea], disponible en:

https://www.inegi.org.mx/programas/cnije/2014/

Censo Nacional de Impartición de Justicia Estatal Censo Nacional de Impartición de Justicia Estatal 2015, [en línea], disponible en: https://www.inegi.org.mx/programas/cnije/2015/default.html\#Tabulados;

Censo Nacional de Impartición de Justicia Estatal 2016, [en línea], disponible en: https:// www.inegi.org.mx/programas/cnije/2016/default.html\#Tabulados

Censo Nacional de Impartición de Justicia Estatal 2017, [en línea], disponible en: https:// www.inegi.org.mx/programas/cnije/2017/

Censo Nacional de Impartición de Justicia Estatal 2018, [en línea], disponible en: https://www.inegi.org.mx/programas/cnije/2018/default.html\#Tabulados

Kala, Julio César (2003), Fenomenología de la Delincuencia. Ciudades Seguras IV, México, FCE.

KaUfman, Michael (1995), Los hombres, el feminismo y las experiencias contradictorias del poder entre hombres [en línea]. Disponible en: www.michaelkaufman.com.

KeIjzer Benno (1997), "El varón como factor de riesgo: masculinidad, salud mental y salud reproductiva”, Esperanza Tuñon (coord.), Género y Salud en el Sureste de México, México, El Colegio de la Frontera Sur / Universidad Juárez Autónoma de Tabasco.

Kimmel, Michael (1997), "Homofobia, temor, vergüenza y silencio en la identidad masculina”, T. Váldes y J. Olavarría (eds.), Masculinidad/es, poder y crisis, Chile, Isis Internacional/ Facultad Latinoamericana de Ciencias Sociales, pp. 49-62.

(2001), "Masculinidades globales: restauración y resistencia", C. Sánchez y J. C. Hidalgo (eds), Masculino Plural: construcciones de la masculinidad, Lleida, Universitat de Lleida, pp. 47-76.

Lombroso, Cesare (1920), Los criminales, Barcelona, Editorial Atlante. 
(1975), Lombroso y la Escuela Positiva Italiana, Madrid, CSdeIC.

Lombroso, Cesare and Ferrero, Guglielmo (2004), Criminal woman, the prostitute and the normal woman, trad., Nicole Hahn Rafter and Maty Gibson, Duke University Press, Durham.

Maqueda, María Luisa (2014), Razones y sinrazones para una criminología feminista, Madrid, Instituto Andaluz Interuniversitario de Criminología/ Dykinson.

NASH, Mary (2004), Mujeres en el mundo. Historia, retos y movimientos, Madrid, Alianza.

Olavarría, José (2006), "Hombres e identidad de género: algunos elementos sobre los recursos de poder y violencia masculina”, G. Careaga y S.Cruz (eds.), Debates sobre masculinidades, México, Programa Universitario de Estudios de Género-Universidad Nacional Autónoma de México, pp. 131-146.

Organización de las Naciones Unidas (onu) / Comisión de la Condición Jurídica y Social de la Mujer, (2013), Informe sobre el $57^{\circ}$ periodo de sesiones, Doc. E/2013/27E/CN.6/2013/11, Nueva York, ONU.

Osborne, Raquel (2009), Apuntes sobre violencia de género, Barcelona, Ediciones Bellatera.

PARK, Robert (1999), La ciudad y otros ensayos de ecología urbana, España, Serbal.

Parsons, Talcott (1984), El sistema social, Madrid, Alianza.

Parsons, Talcott (1972), "La estructura social de la familia", La familia. Fromm, Horkheimer, Parsons, Barcelona, Península, pp. 31-66.

Pitch, Tamar (2003), Un derecho para dos. La construcción jurídica de género, sexo y sexualidad, Madrid, Trotta.

Rodríguez LunA, Ricardo (2018), “La exaltación de lo viril en el positivismo criminológico: la masculinidad en el delincuente del siglo XX”, Alegatos, UAM-Azcapotzalco, núm. 99, mayo agosto, pp. 309-324.

Rubin, Gayle (1989), "Reflexionando sobre el sexo: notas para una teoría radical de la sexualidad”, en Carole S. Vance (comp.), Placer y Peligro. Explorando la sexualidad femenina, Madrid, Hablan las mujeres, pp. 113-190.

Secretaria de Gobernación (2011), "Norma técnica para la clasificación nacional de delitos del fuero común para fines estadísticos”, Diario Oficial de la Federación, México, Secretaria de Gobernación.

SEIDlER, Victor (2000), La sinrazón masculina. Masculinidad y teoría social, México, Paidós.

SMART, Carol (1977), Women, crime and criminology: a feminist critique, London, Routledge \& Kegan Paul. 
(1995), Law, crime and sexuality. Essays in feminism, London, SAGE.

STANKo, Elizabeth (2009), "Es pot reduir la por de la delinqüència que tenen les dones”, Revista Catalana de Seguretat Pública, no. 20, pp. 47-60.

TAYLOR, Ian et. al. (1990), La nueva criminología. Contribución a una teoría social de la conducta desviada, Argentina, Amorrortu.

Thomas, William I. (1899), "Sex in primitive morality", American Journal of Sociology, núm. 4, pp. 774-787.

(1906), "The adventitious character of woman", American Journal of Sociology, núm. 1, pp. 32-44.

Thomas William I. y Znaniecki, Forian (2004), El campesino polaco en Europa y en América, Madrid, Centro de Investigaciones Sociológicas/Agencia Estatal Boletín del Estado.

Unión Europea (2002), Protección de las mujeres contra la violencia. Recomendación Rec (2002) 5 . (2006), Dictamen. La violencia doméstica contra las mujeres. Núm. 2006/C 110/15. (2011), Pacto europeo por la igualdad de género (2011-2020). Bruselas, Unión Europea. Doc. 2011/C 155/o2.

Von Krafft-Ebing, Richard (2009), "Pathological sexuality”, The origins of criminology, a reader, edited by Nicole Rafter, Great Britain, Routledge, pp. 65-73. 